David M. Bond MB BCHIR MA DCH CCFP,

Brian Milne MD M SC FRCPC,

John Pym MB BS FRACs FRCSC, David Sandler MD

\title{
Cardiac tamponade complicating anaes- thetic induction for repair of ascending aorta dissection
}

A case is described of a 69-year-old woman with dissection of the ascending aorta who developed cardiac tamponade during induction of anaesthesia. The tamponade was diagnased by a haemodynamic profile showing approximation of the central venous, pulmonary wedge and pulmonary arterial diastolic pressures, and was treated with rapid surgical intervention and drainage of the haemopericardium. Cardiac tamponade and dissecting aneurysms of the ascending aorta are conditions with contrasting anaesthetic considerations and the problems encountered are discussed.

The anaesthetic considerations for induction of a patient with a dissecting aneurysm of the ascending aorta differ markedly from those of a patient with cardiac tamponade. We describe the development of cardiac tamponade during the induction of a patient with dissection originating in the ascending aorta which to our knowledge has not previously been reported.

\section{Case report}

A 69 -year-old woman weighing $55 \mathrm{~kg}$ was admilted with a one hour history of severe retrosternal chest pain which radiated to her neck, back and arms. This pain was associated with sweating and nausea. Her past history was unremarkable except for long-standing rheumatoid arthritis treated with corticosteroids. Physical examination

\section{Key words}

SURGERY: cardiac, aortic; HEART: tamponade; ANAESTHESIA: cardiac; COMPLICATIONS: aurtic dissection.

From the Departments of Anaesthesiology. Pharmacology \& Toxicology, and Surgery, Queen's University, Kingston, Ontario.

Address correspendence to: Dr. Brian Milne, Department of Anaesthesia, Kingston General Hospital, Kingston, Ontario, K7L 2 V 7. revealed a palpable mass in the suprasternal notch. Systemic arterial blood pressure was $110 / 60 \mathrm{mmHg}$, heart rate 108 beats $\cdot \mathrm{min}^{-1}$ and regular, and respiratory rate $20 \cdot \mathrm{min}^{-1}$. The right radial pulse was diminished compared with the left and was intermittently absent. Carotid pulses were nomnal. Haemoglobin and serum electrolytes were within nomal limits. An electrocardiogram revealed sinus tachycardia. A chest roentgenogram showed widening of the right superior mediastinium and transverse arch of aorta. Computerized tomography showed a mediastinal haematoma and a thin intraluminal membrane in the ascending aorta extending into the arch but not identified at the carinal level in the descending aorta. A diagnosis of dissecting aortic aneurysm originating in the ascending aorta was made and the patient was scheduled for exploration and repair.

A flow-directed balloon-tipped catheter was inserted via the left subclavian vein. Pulmonary arterial pressure was $35 / 15 \mathrm{mmHg}$, pulmonary artery wedge pressure was $10 \mathrm{mmHg}$ and central venous pressure $7 \mathrm{mmHg}$. An arterial line was inserted in the left radial artery. Hydrocortisone $100 \mathrm{mg}$ was given IV. The patient complained of an increase in chest discomfort as she arrived in the OR and there was no change in her clectrocardiogram.

Propranolol was administered in increments to a total dose of $1.5 \mathrm{mg}$, IV. Immediately prior to induction the haemodynamic profile remained unchanged except for a decrease in heart rate to 80 beats $\cdot \mathrm{min}^{-1}$. Anaesthesia was induced with diazepam $1.25 \mathrm{mg}$, fentanyl $2000 \mu \mathrm{g}$ and lidocaine $80 \mathrm{mg}$ IV. Following succinylcholine $100 \mathrm{mg}$ IV the trachea was intubated with a cuffed tube. Anaesthesia was maintained with 100 per cent oxygen, fentanyl, pancuronium and controlled ventilation. Two minutes after intubation and before surgery commenced the pulmonary artery pressure was noted to be $35 / 22 \mathrm{mmHg}$, pulmonary artery wedge pressure $21 \mathrm{mmHg}$ and central venous pressure $18 \mathrm{mmHg}$. This change was associated with a fall in systemic arterial pressure to $85 / 55 \mathrm{mmHg}$. Rapid sternotomy and exploration revealed $200 \mathrm{ml}$ of blood in the percardial space. The blood pressure fell to 
as low as $78 / 60 \mathrm{mmHg}$ despite increased fluid administration. Following drainage of the haemopericardium the systemic arterial pressure rose immediately to $110 / 70$ mmHtg and pulmonary arterial and central venous pressures returned to normal. She was hypotensive for five minutes. The dissection was found to commence $3 \mathrm{~cm}$ above the aortic valve and the coronary ostia were intact. The patient was heparinised and full cardiopulmonary bypass instituted. Under profound hypothermia and circulatory arrest a tie-in graft was placed inside the ascending aorta. Circulatory arrest lasted seven minutes. The aortic valve was resuspended. A sodium nitroprusside infusion was used as required during by-pass to maintain mean arterial pressure between 55 and $75 \mathrm{mmHg}$. Heparinisation was reversed with protamine. The patient was transferred to the surgical intensive care unit for ventilation postoperatively. Her postoperative course was complicated by a right parieto-occipital cerebral infarction which occurred the day following surgery and from which she made a good recovery.

\section{Discussion}

This case demonstrates the developrnent of cardiac tamponade during anaesthetic induction in a patient with a dissecting aneurysm of the ascending aorta; these being conditions with contrasting anaesthetic considerations. In tamponade, an increase in pericardial fluid causes increased pericardial pressure due to the low compliance of the peridarcium. Diastolic filling of the ventricles is impaired causing a rapid increase in ventricular enddiastolic pressure and early closure of the atrioventricular valves. This leads to poor ventricular filling and thus a decreased stroke volume and cardiac output. ${ }^{1}$ Accordingly, management of tamponade involves expansion of the intravascular blood volume and use of a positive inotrope and chronotrope to maintain cardiac filling, contractility and rate ${ }^{2,3}$ In dissection of the ascending aorta, patients are at risk from pressor responses to laryngoscopy and intubation. Besides the possibility of inducing myocardial ischaemia, sympathetically mediated increases in blood pressure and heart rate can lead to further propagation of the dissecting haematoma, or aortic rupture, or both. ${ }^{4,5}$

Despite an uneventfu! induction and an intubation achieved without a cough or rise in blood pressure, the haemodynamic profile changed following intubation to show approximation of the central venous pressure, pulmonary wedge pressure and pulmonary arterial diastolic pressure suggesting the development of cardiac tamponade. $^{2}$ This was associated with relative systemic arterial hypotension which resolved following drainage of the haemopericardium. This course of events would suggest that the chest pain being experienced by the patient on arrival in the operating room was the result of further dissection, which produced a haemopericardium during induction.

Propranolol is used in the treatment of dissection of the ascending aorta for its ability to reduce the shear stress on the aortic walls, thus reducing the tendency to further dissection. ${ }^{6}$ However, the negative inotropic properties of propranolol are undesirable in patients with tamponade. Initial administration of a relatively small dose of propranolol to this patient caused a decrease in heart rate and no significant reduction in blood pressure. It may have contributed to the hypotension secondary to the development of tamponade. In this case, the usc of propranolol as indicated by the pathology present before induction became contraindicated by the tamponade which deyeloped during induction. Propranolol may have impaired the response of the myocardium to the endogenous inotropes which would have been released with the onset of cardiac tamponade. In addition, the institution of positive pressure ventilation in combination with cardiac tamponade may have played a role in the development of hypotension post-induction.

The progression of events in this case illustrates the changes of the development of pathology for which management includes positive chronotropes and inotropes in which a proportion of the $\beta$-receptors in the myocardium are blocked. This serves to re-emphasize the inherent instability of aurtic dissections and the importance of the immediate availability of the surgeon during the management of these patients who can open the chest or place the patient on femoral-femoral bypass if required.

The site chosen for pulmonary artery and arterial catheter insertion should be carefully considered. Insertion of a left subclavian venous catheter could be criticized as hazardous in this patient. Entry of a needle into the adventitial haematoma around an enlarged arch or laterally displaced subclavian artery is potentially disastrous. A high right internal jugular or external jugular approach may have been preferable. Direct arterial pressure should be measured in the left radial artery as aortic dissections arising in the ascending aorta may involve the right subclavian artery, even though all branches of the aorta are potentially vulnerable. ${ }^{5}$

There is a potential for cerebral ischaemia if the aortic dissection involves the aortic arch and the vessels supplying the cerebral circulation. Perhaps the involvement of the aortic arch or the use of circulatory arrest in these patients may constitute an indication for the use of cerebroprotectants, although their benefit is controversial.

In conclusion, we present the development of cardiac tamponade during the industion of a patient with a dissecting aneurysm of the ascending aorta; these being conditions with contrasting anaesthetic considerations. 
Propranolol should be used with caution and the potential of an ascending aortic dissection involving other structures must be borne in mind during management of these patients necessitating the immediate availability of the surgeon.

\section{References}

1 Shabetai R, Fowler NO, Gunteroth WG. The hemodynamics of cardiac tamponade and constrictive pericarditis. Am J Cardiol 1970; 26: 480-9.

2 Lake CL. Anesthesia and pericardial disease. Anesth Analg 1983; 62: 431-43.

3 Kaplan $J A$, Bland $J W$, Dunbar RW. The perioperative management of cardiac tamponade. South Med J 1976; 69 : $417-9$.

4 Dunbar RW. Thoracic Aneurysms. In: Kaplan JA (Ed), Cardiac Anesthesia. Grune \& Stratton 1979: 369-76.

5 Dritz RA. Surgery on the aorta and peripheral arteries. $I n$ : Ream AK, Fogdall RP (Eds), Acute cardiovascular management, unesthesia and intensive care. JB Lippincon Co. 1982: $728-54$.

6 McFarland $J$, Willerson $J-T$, Dinsmore RE et al. The medical treatment of dissecting aortic aneurysms. N Engl J Med 1972; 286: 115-9.

\section{Résumé}

On rapporte le cas d'une femme de 69 ans se présentant pour une dissection de l'aorte ascendante et qui a développé une tamponnade lors de l'induction de l'anesthésie. La tamponnade était diagnostiquée par le profil hêmodynamique qui a démontré une égalisation de la pression veineuse centrale, pression de l'artère pulmonaire bloquée et de la pression diastolique de l'artère pulmonaire. Elle fut traité par une intervention chirurgicale rapide et un drainage de i'hémopéricarde. La tamponnade cardiaque a la dissection de l'anévrysme de l'aorte ascendante sont des conditions qui présentent des considérations anesthésiques opposées. Celles-ci ainsi que les problemes encourus sont discutés. 